Questions vives

\section{Questions Vives}

Recherches en éducation

Vol.4 $n^{\circ} 13$ | 2010

Dispositif didactique ? Dispositif pédagogique?

Situations d'apprentissage !

\title{
La place de l'évaluation dans la réforme du système éducatif marocain : questions pour la recherche
}

Hamadi Akrim, Gérard Figari, Lucie Mottier-Lopez et Mohammed Talbi

\section{(2) OpenEdition}

Journals

Édition électronique

URL : http://journals.openedition.org/questionsvives/323

DOI : 10.4000/questionsvives.323

ISBN : 978-2-8218-1086-0

ISSN : $1775-433 \mathrm{X}$

Éditeur

Université Aix-Marseille (AMU)

Édition imprimée

Date de publication : 1 janvier 2010

Pagination : 387-400

ISBN : 978-2-912643-37-7

ISSN : $1635-4079$

\section{Référence électronique}

Hamadi Akrim, Gérard Figari, Lucie Mottier-Lopez et Mohammed Talbi, «La place de l'évaluation dans la réforme du système éducatif marocain : questions pour la recherche », Questions Vives [En ligne], Vol. $4 \mathrm{n}^{\circ} 13$ | 2010, mis en ligne le 01 janvier 2011, consulté le 10 décembre 2020. URL : http:// journals.openedition.org/questionsvives/323; DOI : https://doi.org/10.4000/questionsvives.323

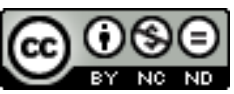

Questions Vives est mis à disposition selon les termes de la licence Creative Commons Attribution -

Pas d'Utilisation Commerciale - Pas de Modification 4.0 International. 


\title{
La place de l'évaluation dans la réforme du système éducatif marocain : questions pour la recherche
}

\author{
Hamadi AKRIM 1 \\ Gérard FIGARI2 \\ Lucie Mottier LOPEZ ${ }^{3}$ \\ Mohammed TALBI ${ }^{4}$
}

Résumé: Existe-t-il des modèles applicables à la définition d'une politique d'évaluation accompagnant la réforme d'un système éducatif ? Cet article propose de répondre à cette question en l'appliquant au système marocain d'éducation et de formation. A travers la littérature existante, il formule, à la fois, la problématique de l'évaluation des dispositifs et des programmes dans une perspective aussi bien stratégique que méthodologique et celle de l'évaluation des apprentissages en concordance avec l'introduction de l'approche par compétences. L'intérêt de cette revue réside dans le fait de contribuer à la recherche de modèles d'évaluation pouvant aider au choix d'une politique évaluative au niveau des micro et méso-structures.

Mots-clés: Réforme du système éducatif, modèles d'évaluation, politique d'évaluation, évaluation des dispositifs, évaluation des apprentissages.

Abstract: Are there models for defining a policy assessment to accompany the reform of the education system? This article aims at examining this question through the Moroccan system of education and training. The evaluation of programs and devices is discussed from a strategic and methodological perspective. The assessment of students' learning is also explored according to the implementation of the "Competency-based Approach". The interest of this review is to contribute to defining assessment models that could help to choose a policy-level evaluation with regard to micro and meso-structures.

Keywords: Educational reform ; assessment models ; evaluation of policy; evaluation of learning devices ; assessment of learning.

\footnotetext{
${ }_{1}$ Professeur - Centre Pédagogique Régional de Casablanca, Maroc.

2 Professeur émérite - Université P. Mendes France Grenoble, France.

3 Professeur - Université de Genève, Suisse.

4 Professeur - Université Hassan II Mohammedia-Casablanca, Maroc.
} 
Hamadi Akrim, Gérard Figari, Lucie Mottier Lopez, Mohammed Talbi

La place de l'évaluation dans la réforme du système éducatif marocain : questions pour la recherche

\section{Introduction}

La réforme du système éducatif au Maroc, lancée au début du 21ème siècle, visant à élever le niveau des élèves et à améliorer la qualité de l'enseignement et des apprentissages, appelle un développement considérable des activités d'évaluation pour répondre aux nécessités de pilotage qu'elle entraîne.

Certes, l'évaluation s'inscrit, traditionnellement, dans une perspective de jugement et de prise de décision, à propos des apprentissages, des enseignements ou des programmes. Mais elle reste spécifique à un contexte historique et sociétal et elle constitue, toujours, un reflet des objectifs explicites ou implicites d'un « projet ». C'est vrai, en général et c'est vrai, aussi, pour l'éducation. C'est ainsi que la recherche spécialisée sur l'évaluation éducative a montré, depuis les travaux fondateurs de Tyler (1967) jusqu'aux études les plus récentes (Shadish et al., 1991) et actuelles (Figari \& Mottier Lopez, 2006), la variété et la spécificité des modèles d'évaluation conçus pour s'adapter aux différentes problématiques contextuelles et situationnelles. Dans la continuité de ces travaux, on peut dire que l'importance des enjeux de la réforme marocaine constitue une invitation à réfléchir sur les choix scientifiques possibles pour nourrir et étayer une politique d'évaluation en cours d'élaboration dans un tel contexte.

Quelles questions pose à la recherche la conception d'un dispositif d'évaluation accompagnant un projet éducatif consistant à revaloriser la place de l'élève au centre du système en restructurant ses dispositifs et à unifier les approches pédagogiques autour de l'acquisition de compétences?

La somme d'études et de recherches consultables sur ce thème est considérable. Aussi n'est-il pas concevable, ici, de prétendre en rapporter l'exhaustivité. C'est pourquoi, après avoir formulé les principales problématiques d'évaluation pouvant émerger du contexte marocain (\$1), l'ambition de cet article consistera à mobiliser quelques concepts et modèles jugés utilisables pour réfléchir sur le sujet à partir de l'évocation des travaux de la première session d'études de la section marocaine associée à l'ADMEE-Europe (juin 2009). On se limitera donc à repérer plus particulièrement les questions relatives à l'évaluation des dispositifs (§ 2) et à l'évaluation des apprentissages et des compétences (§ 3) ayant donné lieu à des synthèses présentées par les auteurs.

\section{Emergence de questions d'évaluation dans la réforme d'un système éducatif : l'exemple marocain}

\section{Une réforme}

La réforme d'un système éducatif constitue toujours un objet de débat, politique d'abord (comme réponse à des besoins profonds d'une société et d'une jeunesse en mutation, dans un contexte international particulièrement prégnant), mais aussi pédagogique (comme changement des pratiques et comme développement de nouvelles compétences). 
Dispositif pédagogique ? Dispositif didactique? Situations d'apprentissage !

C'est le cas du Maroc, actuellement, avec la "Charte Nationale d'Education et de Formation " $(\mathrm{CNEF})^{5}$. En effet, la réforme qu'elle a instituée vise, à la fois, la généralisation de l'enseignement fondamental (pour répondre à la pression de la logique socioéconomique dominante) et l'amélioration du fonctionnement des apprentissages en plaçant l'apprenant au centre de l'action pédagogique et en développant une « approche par compétences ».

\section{Des diagnostics}

La Direction de l'Evaluation du Système Educatif (DESE) a mené plusieurs évaluations, notamment par des tests transnationaux du genre TIMMS-R, MLA, CTL, PIRLS ${ }^{6}$, afin de percevoir les acquis des élèves de niveaux comparables.

II ressort de ces études que :

- le niveau des élèves marocains reste nettement en dessous de la moyenne internationale et régionale?. Caractérisé par la grande disparité entre milieux et entre sexes, le système d'éducation se retrouve comme handicapé par cet écart des niveaux, économique et social, qui se répercute de façon négative sur le rendement scolaire détecté par les instances internationales ;

- $\quad$ la réussite à l'Université est conditionnée par la capacité des étudiants à s'adapter au mode d'enseignement dont la langue d'enseignement pour les matières scientifiques qui devient le Français au lieu de l'Arabe au secondaire. A titre d'exemple, depuis 1988 environ, le taux d'échec en première année universitaire a varié entre $66 \%$ et $75 \%$, alors que $52 \%$ des étudiants ne dépassaient pas le premier cycle universitaire et 60\% abandonnaient avant la licence ; 10\% seulement obtenaient celle-ci en quatre années prévues dans l'ancien système (Akrim, ElJamali, El-Bousaadani, Radid, \& Talbi, 2006). D'autre part, en dépit de l'avantage des contrôles continus instaurés par le système LMD, les pratiques évaluatives restent tributaires d'utilisations non essentiellement fondées sur une méthodologie propre à spécifier les contenus et à concevoir les référentiels d'évaluation ${ }^{8}$.

Les résultats des enquêtes comparatives internationales montrent que le système d'éducation et de formation marocain reste en deçà des espérances ${ }^{9}$. II demande :

- $\quad$ une ingénierie plus efficace permettant d'augmenter la quantité et améliorer la qualité des intrants dans le système éducatif, tel les ressources humaines, les curricula et enseignements ainsi que le financement et l'administration ;

- $\quad$ des incitations des intervenants dans l'action éducative alignées sur les résultats à travers un suivi, une évaluation, une motivation et une récompense ;

\footnotetext{
${ }^{5}$ La Charte Nationale d'Education et de Formation (CNEF) est éditée en 1999 par la Commission Spéciale Education et Formation [http://www.men.gov.ma]

6 TIMSS-R : Trends In International Mathematics and Science Study- Repeat

$M L A$ : Monitoring learning achievement - suivi régulier des acquis scolaires ;

CTL : Conditions of teaching and learning;

PIRLS : Programme international de recherche en lecture scolaire.

7 [http://www.unesco.org/education/wef/countryreports/marocco/rapport_1.html] (consulté le 13/10/09)

8 D'après des enquêtes réalisées au sein de l'ORDIPU.

9 [http://siteresources.worldbank.org/INTMENA/Resources/EDU_Summary_FRE.pdf]
} 
Hamadi Akrim, Gérard Figari, Lucie Mottier Lopez, Mohammed Talbi

La place de l'évaluation dans la réforme du système éducatif marocain : questions pour la recherche

- $\quad$ des mesures favorisant une plus grande responsabilité publique permettant à tous les intervenants (parents, élèves et autres) d'exercer une influence sur la politique éducative, à tel point qu'ils peuvent la modifier dans le sens d'une amélioration.

Par ailleurs, de manière générale, les pratiques évaluatives semblent en retard par rapport aux orientations affichées par les multiples changements qui affectent le système d'enseignement. C'est le cas de l'approche par compétences, théoriquement instaurée avec la réforme, et qui manque d'outils d'accompagnement et d'une méthodologie d'évaluation spécifique.

L'ensemble de ces diagnostics, appuyés par le rapport du CSE sur l' "Etat et Perspectives du Système d'Education et de Formation 10 ", a justifié la relance de la réforme par le Plan d'Urgence ${ }^{11}$ qui fixe des objectifs ambitieux justifiant la mise en place d'une politique d'évaluation adaptée aux enjeux en présence. D'où l'intérêt de la réflexion dont veut rendre compte cet article.

\section{Vers une politique d'évaluation}

Dans ce contexte, l'établissement de nouvelles modalités d'évaluation est à l'ordre du jour, et, à tous les niveaux du système d'éducation et de formation, il est question de démarche qualité, d'efficacité, d'efficience, de performance, de taux de diplomation, etc. Toutes ces expressions font allusion à l'évaluation, repère significatif de toute réforme du système éducatif qui cherche une signification à la prise de décision et à ses résultats.

Une politique d'évaluation du système est en effet en place: la création de l'Instance Nationale de l'Evaluation du Système d'Education et de Formation (INESEF), organe du Conseil Supérieur de l'Enseignement (CSE), dont la mission consiste à procéder à des évaluations globales, sectorielles ou thématiques, permet un espace de proposition, d'échange et d'expérimentation. Elle fait appel à la recherche scientifique : elle soutient les activités de recherche dans ce domaine ${ }^{12}$.

\section{Des questions pour la recherche}

Parmi les questionnements qui émergent des manifestations récentes autour du thème de l'évaluation dans le système éducatif au Maroc, deux semblent particulièrement prégnants.

- Le premier est relatif à l'évaluation des dispositifs et des programmes, depuis l'enseignement préscolaire jusqu'à l'Université. Témoins de cette préoccupation, les colloques organisés par l'ORDIPU13 comme celui intitulé «Place de l'évaluation

\footnotetext{
10 Le rapport du CSE, édité en 4 volumes en 2008, retrace, chiffres à l'appui, le bilan contrasté des avancées et des dysfonctionnements au sein du système. Royaume du Maroc, CSE, Rapport annuel 2008.

${ }^{11}$ Le Plan ou Programme d'urgence élaboré en 2008 vise à accélérer la réforme et corriger le bilan mitigé des réalisations accomplies: [http://www.men.gov.ma]

${ }^{12}$ Article 13 du Bulletin Officiel [http://www.sgg.gov.ma/BO/bulletin/Fr/2006/BO_5396_fr.pdf].

13 L'Observatoire de Recherche en Didactique et Pédagogie Universitaire fait parti de l'Université Hassan II de Mohammedia-Casablanca, il regroupe plusieurs équipes et laboratoires de recherche de différents établissements de l'enseignement supérieur et de la formation des cadres.
} 
Dispositif pédagogique ? Dispositif didactique? Situations d'apprentissage !

dans la stratégie de développement du système éducatif: bilan préliminaire, évaluation et perspective de la réforme LMD " (octobre 2007). Les préoccupations, lors de ce colloque, concernent l'évaluation des enseignements par les étudiants et la prise en compte de leurs points de vue et de manière plus générale, l'évaluation du dispositif d'enseignement et de formation au sein du système LMD ;

- Le second intéresse l'évaluation des apprentissages dans la perspective de l'introduction volontariste d'une approche pédagogique par compétences.

Des événements scientifiques ont révélé également l'existence de cette préoccupation chez les acteurs éducatifs, mêlant souvent ce thème au précédent. Citons, par exemple, la première rencontre annuelle organisée en 2008 par l'INESEF sur « ''évaluation des systèmes d'éducation et de formation dans tous ses états ". Le but de ce colloque est d'imprégner l'ensemble des acteurs du secteur de l'éducation et de la formation, d'une culture de l'évaluation, et de pourvoir un espace de réflexion sur des pratiques d'évaluation, pertinentes et adaptables aux particularités du contexte marocain. Elle s'articule autour des axes : pratiques de l'évaluation en éducation; culture, acteurs, méthodologies et finalités de l'évaluation; le métier d'évaluation : profil et exigences de la pratique du métier de l'évaluation; ; l'évaluation de la réussite scolaire.

Ces deux questionnements intéressent la recherche en éducation: ils contribuent à nourrir deux axes scientifiques majeurs organisant, chacun, des mouvements de pensée et une littérature spécifiques dont un aperçu peut être donné ici. Ils vont être abordés, non en tant que thèmes à développer mais comme "problématiques" de recherche dans le domaine de l'évaluation éducative.

Qu'est-ce qu'une problématique d'évaluation? Ce n'est pas seulement une question à développer, issue d'un besoin ou du bon sens. II s'agit d'une démarche scientifique consistant en une transformation de l'interrogation initiale (quelle évaluation pour accompagner une politique?) en questionnement scientifique (quels modèles et quelles conceptualisations utiliser pour choisir une approche évaluative adaptée au contexte concerné, ici le système éducatif marocain en mutation).

La question qui se pose est donc de chercher à définir la contribution que la recherche peut y apporter. II s'agit, en effet, d'examiner les deux sortes de problématiques d'évaluation apparemment majeures émergeant de la situation qui vient d'être décrite.

\section{Les problématiques d'évaluation des dispositifs}

La première série de problématiques évoquée concerne la dimension institutionnelle (programmes, pilotage, dispositifs). Ces problématiques sont non seulement stratégiques (analyse des résultats obtenus par le système : enquêtes internationales de type PISA dans les pays de l'OCDE ou dirigées par l'UNICEF ou L'UNESCO et nationales qui restent locales (menées par des équipes de recherches universitaires) ou à caractère institutionnel (ordonnées par le département ministériel concerné), mais aussi scientifiques (critique et reconstruction des référentiels ainsi que production de méthodologies fondées et utilisables par les acteurs).

Deux types de problématiques générales, stratégiques et méthodologiques, adaptées à la situation, apparaissent dans la littérature sur l'évaluation des dispositifs. 
Hamadi Akrim, Gérard Figari, Lucie Mottier Lopez, Mohammed Talbi

La place de l'évaluation dans la réforme du système éducatif marocain : questions pour la recherche

\section{Des problématiques stratégiques}

Deux questionnements précis précèdent, en général, les évaluations de type « institutionnel »:

Le premier consiste à étudier l'objet à évaluer : une politique d'évaluation appliquée à des dispositifs, des programmes, des curricula. Comment définir et circonscrire cet objet complexe qui constitue pourtant le lien entre le macrosystème et le microsystème: une mésostructure au service d'une finalité nationale mais destinée à agir sur un contexte local ? On va garder, ici, comme dénominateur commun à toutes les formes de mésostructures la notion de "dispositif " car elle constitue une entité isolable, observable, comparable et qu'elle est utilisée pour matérialiser les leviers des politiques publiques. C'est donc à une théorie du dispositif éducatif (et à sa conceptualisation) qu'on fera appel avant de mettre en place tout appareil d'évaluation. Pourra-t-on, alors, aboutir à une modélisation du dispositif ?

L'initiateur du concept, M. Foucault, définit le dispositif comme un « réseau que l'on peut établir entre les éléments " (1994, p.299). Ce concept a servi à traiter la dimension technique de certains phénomènes sociaux comme la pédagogie ou les politiques sociales, dans « une logique de moyens mis en œuvre en vue d'une fin » (Peeters \& Charlier, 1999).

Un dispositif est, à la fois, un objet concret (observable : alternance, formation à distance, formation continue des enseignants...) et une abstraction (découpage de phénomènes donnant lieu à une représentation précise et à une explication de leurs régularités : dispositif de lutte contre l'échec scolaire, dispositif d'ascenseur social...). Pour l'appréhender (le concevoir, l'observer, le réguler, l'évaluer...), il est utile de pouvoir disposer de conceptualisations (sous forme de terminologies, de théorisations ou de modélisations) qui le spécifient. Ce n'est pas, ici, le lieu d'en faire l'inventaire exhaustif, mais on peut s'appuyer sur certaines d'entre elles pour étayer une catégorisation des principaux descripteurs du " dispositif ", qui ont été réduits, en la circonstance, aux quatre suivants : une alternative à la pédagogie "magistrale " (Montandon, 2002), une "mise en situation " pour la formation (Altet, 1997), un mécanisme "éducatif " (Figari, 1994), une approche technique des environnements quotidiens (Peeters \& Charlier, 1999). Ces derniers auteurs réalisent une synthèse de travaux proposant les contours d'une « théorie du dispositif » dont on peut lire une synthèse dans l'article consacré à une synthèse de travaux sur l'évaluation des dispositifs dans le numéro spécial des 30 ans de la revue Mesure et évaluation en éducation (Figari, 2008). On peut, simplement, ici, à partir des travaux de Peeters et Charlier (1999), en retenir les caractéristiques les plus parlantes (citées dans l'article pris en référence) :

- le concept de "dispositif » peut être défini comme un "entre deux ", dans la mouvance post-moderne, figure intermédiaire entre une approche totalisante (structure) et une approche complexe (interactions). On peut lui attribuer une rationalité instrumentale en le considérant comme la concrétisation d'une intention au travers de la mise en place d'environnements aménagés (au moyen de mécanismes finalisés constituant des instruments pour l'action);

- le dispositif constituerait l'intermédiaire entre l'objectif et le résultat d'une activité en visant essentiellement son efficacité. En cela, il deviendrait un «instrument de projet " ;

- enfin, il définirait un " espace de médiation " : un espace de jeu (avec ses règles et ses stratégies) pour les acteurs impliqués dans un même projet. II régulerait le 
Dispositif pédagogique ? Dispositif didactique? Situations d'apprentissage !

fonctionnement de ce dernier en constituant un instrument de médiation entre sujet et objet, entre objectifs et résultats.

Plutôt que d'une « théorie » du dispositif, nous disposons, avec les apports de Peeters et Charlier, d'une conceptualisation de cette notion et d'une logique attachée à son emploi : plus qu'un objet isolé et indépendant, le dispositif serait un processus d'intervention sur le monde, au service d'un projet humain, un "entre deux " indispensable à la médiation entre sujet (auteur de l'intentionnalité), objet (contenu de l'action) et technique (ensemble des instruments).

Le second questionnement concerne l'évaluation elle-même: objet fréquent de malentendus et de critiques ainsi que de combats d'écoles, l'évaluation en éducation et formation mérite une clarification avant d'être instituée et mise en pratique.

Quelles formes peut prendre un processus évaluatif ? Quelles typologies invoquer pour situer et caractériser une politique naissante d'évaluation du (et dans le) système éducatif ? S'il s'agit, par exemple, d'impact à apprécier, convient-il de s'inscrire dans les grandes catégories internationales (utilisées notamment par la Commission Européenne) qui distinguent entre évaluation ex/ante (analyse prédictive), intermédiaire (appelée aussi concomitante, à mi-parcours), et enfin, ex/post (production de résultats, en ne considérant que des objets standardisés à mesurer et à comparer) ?

Mais s'il s'agit d'accompagner un changement, d'améliorer le fonctionnement d'un dispositif ou de développer sa qualité, les modèles d'évaluation ne seront-ils pas à chercher :

- dans «l'assurance qualité " (OCDE, 2008)? autour de la définition d'un " processus visant à ce que les parties prenantes soient certaines que l'offre (moyens mis en œuvre, processus et résultats) répond aux attentes et est à la hauteur des exigences minimums ";

- dans les théories autour de la gouvernance (par exemple, les modèles de pilotage, chez de Landsheere (1994)?

\section{Des problématiques méthodologiques}

Toute évaluation se manifeste par un appareil méthodologique de mesure et d'appréciation. Le postulat majeur qui inspire la réflexion engagée ici est que cet appareil ne constitue que la partie émergente de l'iceberg, le reste, profond et majoritaire, étant constitué par la dimension précédente (c'est-à-dire la problématique stratégique). Comment l'objet à évaluer (une politique d'évaluation) étant ainsi défini et modélisé, va-t-il être observé et par qui ? Trois approches concurrentes (ou complémentaires), selon Rodrigues (2006, p.194), se présentent alors au choix des évaluateurs :

- une approche externe, "objectiviste " qui évaluera les dispositifs selon des standards externes et comparatifs ;

- $\quad$ une approche interne, "subjectiviste ", qui privilégiera l'autoévaluation ;

- une approche interactive, "dialectique " qui aura comme orientation de favoriser une attitude participative des acteurs dans le but d'une recherche collective de sens. 
Hamadi Akrim, Gérard Figari, Lucie Mottier Lopez, Mohammed Talbi

La place de l'évaluation dans la réforme du système éducatif marocain : questions pour la recherche

Dans tous les cas, la question du choix des critères et des indicateurs de l'évaluation se pose aux évaluateurs qui adoptent, explicitement et le plus souvent implicitement, une référentialisation quelconque : il s'agira du recours à des référentiels existants (système de normes ou de standards, référentiels institutionnels ou professionnels, etc.). A moins que la référentialisation ne soit assumée par les évaluateurs. Cela peut être le cas si la politique de pilotage et d'évaluation du dispositif privilégie l'implication des différents acteurs du dispositif dans son évaluation. L'intention de l'évaluateur consiste alors à favoriser la participation de ces derniers à l'amélioration et à une plus grande efficacité du dispositif.

On voit bien que le sens d'une évaluation va être influencé par le choix de ses procédures. Celles-ci reflétant des modèles sous-jacents d'évaluation qui les inspirent, les évaluateurs ont tout intérêt à les soumettre à analyse et à remise en question : les ressources de la recherche sont à leur disposition.

\section{Les problématiques didactiques et la question des compétences}

La deuxième série de problématiques concerne les pratiques d'évaluations internes et externes des apprentissages des élèves considérant les orientations didactiques promues par le système éducatif.

\section{Evaluation des apprentissages et des compétences par les enseignants}

Conformément à un mouvement international, le choix de la réforme du système éducatif marocain est de promouvoir une "approche par compétences ", perçue comme offrant les conditions à un apprentissage plus "actif » et "significatif » des élèves. Les arguments classiques de l'approche par compétences sont avancés, notamment l'opposition entre la restitution mécanique de connaissances mémorisées (qualifiée de connaissances "inertes " déjà au début du XXème siècle par Whitehead, 192914) et l'utilisation des ressources apprises dans des situations complexes et inédites. Un accent est mis sur la notion de " compétence transversale " au sens de Pallascio et Beaudry (2000) dans le contexte de l'école québécoise. La réforme, par cette notion, signifie qu'elle promeut l'idée que les élèves doivent être capables de mobiliser des ressources qui transcendent les frontières disciplinaires.

Le bilan des huit ans de la réforme montre que l'approche par compétences reste marginale dans les classes. Plusieurs raisons sont évoquées: la "méthodologie » de l'approche par compétences n'est pas réellement maîtrisée par les enseignants, les programmes d'enseignement sont trop chargés et ils ne sont pas adaptés à cette approche, la formation des enseignants n'a pas été pensée en conséquence. Mais un des freins importants constatés est la difficulté d'une évaluation en actes des compétences, c'est-à-dire dépassant les prescriptions diffusées par des guides préexistants qui prennent peu en

\footnotetext{
14 Whitehead définit les connaissances inertes (inert knowledge) comme étant les connaissances que les individus parviennent à restituer lorsqu'on le leur demande explicitement, mais qu'ils ne parviennent pas à utiliser spontanément dans des situations de résolutions de problèmes. Le débat sur l'enjeu de la mobilisation des connaissances en situation est un débat de longue date, préexistant aux approches par compétences.
} 
Dispositif pédagogique ? Dispositif didactique? Situations d'apprentissage !

considération la culture, les pratiques et les contraintes contextuelles propres au système éducatif marocain.

Le programme d'urgence mis en place suite à ces constats ne peut faire l'impasse d'une réflexion sur un ensemble de points. Au plan didactique et de l'évaluation des apprentissages des élèves, il paraît impossible d'implanter une approche pédagogique innovante sans une réflexion approfondie sur les modalités d'évaluation pour que les praticiens se l'approprient. En effet, si les contenus à enseigner/apprendre ne sont pas évaluables, il y a une forte probabilité que les enseignants les abandonnent.

Si la réforme choisit de promouvoir l'approche par compétences, une réflexion didactique est donc indispensable sur non seulement les situations d'apprentissage des compétences mais également sur les situations de leur évaluation formative et certificative. Autrement dit, évaluer des compétences nécessite une «ingénierie didactique " à propos des types de tâches et des contraintes et ressources externes mises à la disposition des apprenants, à propos de l'articulation entre les tâches complexes (à des fins de mobilisation) et spécifiques (à des fins d'apprentissage des ressources à la mobilisation des compétences), à propos de la temporalité des situations d'apprentissage et à l'évaluation des parcours de progression des élèves. Allal (2007), par exemple, définit plusieurs principes de construction d'épreuves certificatives visant une évaluation authentique et située des compétences de l'élève, dont: faire porter l'évaluation sur une situation qui mobilise plusieurs compétences prioritaires, découper le dispositif d'évaluation en plusieurs étapes qui peuvent comporter des régulations formatives, intégrer des outils et éventuellement des formes d'interaction sociale au fil des étapes. Ce faisant, l'évaluation certificative n'est plus pensée comme étant disjointe de la situation d'apprentissage. Elle est vue comme pouvant contribuer à la consolidation des compétences en tant que prolongement de la situation d'apprentissage. Ces propositions, si on y adhère, demandent la construction de nouvelles représentations de l'évaluation des apprentissages des élèves et de l'interprétation des résultats par les enseignants. Comme développé dans Mottier Lopez (2010), la séparation entre apprentissage et évaluation s'amoindrit, la temporalité de l'évaluation certificative est plus longue (ce qui pose moins de problèmes car elle est vue comme susceptible de produire des apprentissages), l'opposition classique entre évaluations certificative et formative est remise en question (l'évaluation certificative intègre, par exemple, des démarches formatives en amont et en aval de la « note " certificative).

La réforme concerne l'ensemble des ordres d'enseignement (primaire, secondaire, universitaire, professionnel). La notion de compétence représente une unité commune de structuration des plans d'études et des pratiques enseignantes. Si ce choix vise à garantir une certaine cohérence du curriculum, un enjeu est de mieux appréhender les spécificités et contraintes propres à chaque ordre d'enseignement. Par exemple, est-il vraiment pertinent de parler de compétence dans l'éducation préscolaire et dans les premiers degrés du primaire? La notion de compétence peut-elle se définir et s'évaluer de façon similaire dans l'enseignement obligatoire et dans l'enseignement professionnel qui, lui, a une finalité plus explicite par rapport à des pratiques sociales de référence autres que scolaires? Cela semble peu probable.

Perrenoud (1993), parmi différents facteurs faisant échouer une réforme scolaire, identifie le fait d'ignorer la réalité du travail des enseignants. Autrement dit, promouvoir l'enseignement et l'apprentissage de compétences exige non seulement que l'on sache 


\author{
Hamadi Akrim, Gérard Figari, Lucie Mottier Lopez, Mohammed Talbi \\ La place de l'évaluation dans la réforme du système éducatif marocain : \\ questions pour la recherche
}

comment évaluer de façon formative et certificative ces compétences mais également de pouvoir mettre en œuvre ces nouvelles modalités compte tenu des multiples contraintes du terrain et facteurs existants. Pensons par exemple au temps à disposition, aux ressources matérielles, aux contenus des programmes, aux valeurs personnelles et professionnelles des enseignants, aux attentes des parents et des employeurs, à la culture des écoles et des ordres d'enseignement, aux stratégies de pilotage choisies par le système éducatif, etc.

Ce dernier point amène à une réflexion critique qui doit être également menée sur un plan culturel. Non seulement il convient de se demander dans quelle mesure les acteurs concernés par la réforme sont convaincus par l'innovation qui leur est demandée, mais, plus fondamentalement, il importe de mettre en mots leurs perceptions de cette innovation qui est le produit de modèles occidentaux dominants. Une appropriation authentique passe certainement par des adaptations et des modifications significatives. Plutôt que de tenter de les limiter, voire de les regretter, une posture de recherche demande de les examiner avec attention, et de les appréhender comme de réelles potentialités de développements scientifiques.

Par rapport aux différents éléments que nous venons de mentionner, des pistes existent bien évidemment dans la littérature de recherche (voir par exemple, Allal, 2007 ; Rey, Carette, Defrance \& Kahn, 2006 ; Roegiers, 2001 ; Scallon, 2004). De nombreux points restent cependant à investiguer au plan scientifique. La situation actuelle du système éducatif marocain, compte tenu notamment de la volonté politique d'agir sur les différents plans micro et macro-contextuels, est certainement un terrain propice pour des investigations scientifiques. Plusieurs dérives sont néanmoins à éviter. II s'agit de ne pas tomber dans une forme d'hégémonie de l'approche par compétences (ou de ce que l'on peut appeler du " tout compétences "), consistant à nier les différents types d'apprentissages qui sous-tendent les trajectoires de développement des élèves. II s'agit également de déconstruire des fausses oppositions trop souvent affirmées, par exemple entre apprentissage et compétence (alors que l'apprentissage englobe la compétence), entre connaissances et compétences (être compétent exige de posséder des connaissances, entre autres ressources internes et externes à apprendre), ou encore entre situations complexes et spécifiques (ces situations sont complémentaires pour apprendre les ressources et apprendre à les mobiliser). Une autre dérive serait de penser qu'il n'y aurait qu'une seule pédagogie à promouvoir, par exemple la "pédagogie de l'intégration ", instituée par la formation " d'experts » au niveau central, et actuellement en expérimentation dans deux académies aux niveaux des enseignements primaire et secondaire collège. Un enjeu important du plan d'action en faveur de la réforme est d'amplifier la professionnalisation des enseignants, afin qu'ils soient aptes à mettre en œuvre des approches différenciées dans leur classe, tout en portant un regard réflexif et critique sur celles-ci.

\title{
Evaluations externes des apprentissages des élèves
}

La partie précédente s'est préoccupée des pratiques évaluatives des enseignants, c'està-dire des évaluations internes à leur classe ou à leur équipe pédagogique. L'évaluation externe des apprentissages, quant à elle, est conçue par une personne extérieure à la relation didactique qui lie l'enseignant et ses élèves, par exemple par des chercheurs qui ont pour mandat de construire des épreuves standardisées. Ntamakiliro et Longchamp (2008) 
Dispositif pédagogique ? Dispositif didactique? Situations d'apprentissage !

rappellent la double finalité des évaluations externes constatée dans les systèmes éducatifs. D'une part, les évaluations externes sont des compléments aux évaluations diagnostique, certificative ou pronostique réalisées par les enseignants. D'autre part, elles contribuent au pilotage du système scolaire. Les statistiques des résultats des élèves aux épreuves standardisées fournissent des informations, entre autres, sur le degré d'efficacité et d'équité de l'enseignement.

Au sujet de la première finalité, l'intérêt des épreuves externes est de fournir une information aux enseignants qui peut être mise en relation avec une population d'élèves du même âge, mais dépassant largement le cadre de leurs classes. Dans une fonction diagnostique, l'épreuve externe est passée plutôt en début d'année scolaire, les informations sont alors exploitables par l'enseignant pour les élèves qui ont effectué l'épreuve (régulation de premier niveau, selon Allal \& Schwartz, 1996). Dans une fonction certificative, les informations produites par l'épreuve externe réalisée en fin d'année scolaire peuvent être utiles à l'enseignant, par exemple pour ajuster son enseignement l'année suivante (régulation de deuxième niveau).

A propos de la deuxième finalité des épreuves externes, l'agrégation des informations sur les acquis des élèves est utilisée à des fins de stratégies politiques et de régulation du système éducatif. Dans ce cas, la régulation se réalise sur une durée temporelle plutôt longue. Elle concerne souvent d'autres aspects, en plus des activités d'enseignement et d'apprentissage en classe. Par exemple: décision d'allocation de ressources supplémentaires pour certains établissements, conception de nouveaux modules de développement professionnel destinés aux enseignants, élaboration de directives ou de recommandations pour les directions d'école et les conseillers pédagogiques.

\section{Un enjeu : l'articulation entre les évaluations internes et externes}

Alors que la réforme du système éducatif marocain a pour intérêt de vouloir agir sur les pratiques d'enseignement en classe et à la fois sur sa politique de pilotage, un enjeu majeur est d'examiner avec attention l'articulation entre les évaluations externes des acquis des élèves et les pratiques d'évaluation interne des enseignants. La littérature de recherche a mis en évidence un ensemble d'effets produits par la standardisation des épreuves sur les pratiques d'enseignement et le curriculum enseigné. Hargreaves (2003), cité par Maroy (2009), souligne notamment un rétrécissement des choix curriculaires, une centration sur le contenu et la forme des épreuves standardisées (teach to test) au détriment d'autres modalités d'apprentissage et d'évaluation, un alourdissement de la charge du travail administratif des enseignants préjudiciant par exemple le travail en équipe pédagogique, et plus généralement un risque de déprofessionnalisation du corps enseignant. Lorsque les épreuves externes ont un poids important sur les certifications et les décisions d'orientation qui affectent les parcours scolaires des élèves ${ }^{15}$, ces effets risquent de se renforcer. L'étude de Lafontaine, Soussi et Nidegger (2009) montre, par contre, que des évaluations externes à visée diagnostique, dans le cadre d'un pilotage dit "bienveillant ", sont perçues de façon plutôt positive par les enseignants. Elles les engagent dans une dynamique de changement.

\footnotetext{
${ }^{15}$ C'est-à-dire dans le cas d'enjeux importants (high stakes, en anglais).
} 


\author{
Hamadi Akrim, Gérard Figari, Lucie Mottier Lopez, Mohammed Talbi \\ La place de l'évaluation dans la réforme du système éducatif marocain : \\ questions pour la recherche
}

Une réflexion approfondie est donc à mener sur les fonctions à prioriser des épreuves externes.

Le choix du système éducatif de promouvoir l'approche par compétences exige qu'il puisse proposer des épreuves externes adaptées. Les tests standardisés classiques ${ }^{16}$ n'offrent pas les conditions à une évaluation par situations complexes et "authentiques". Des outils alternatifs doivent être développés, tout en considérant la contrainte d'une évaluation externe qui doit pouvoir être appliquée à un grand nombre d'élèves et qui conduit à une agrégation possible des résultats (Direndonck \& Fagnant, 2010). Mais un risque à éviter, quelle que soit la forme de l'épreuve externe d'ailleurs, est que la logique statistique se substitue à la logique didactique. La réflexion didactique et les enjeux d'apprentissage dans le quotidien des classes et des écoles doit rester au centre des préoccupations. Un enjeu est que les résultats des évaluations externes prennent sens pour les problématiques d'apprentissage et d'enseignement et à la fois pour les stratégies de formation et de gestion politique du système éducatif.

Le contexte national, aussi bien que régional ou international, est propice à des démarches d'investigation scientifique afin de questionner de façon critique les évaluations internes et externes des apprentissages des élèves et leur articulation:

- $\quad$ Les pratiques d'évaluation des enseignants et les épreuves externes standardisées sont-elles en adéquation avec les recommandations didactiques rattachées à l'approche par compétences? Quels sont les freins ? Quel équilibre est à construire entre l'évaluation par des tâches complexes et aussi par des tâches spécifiques?

- Quel degré de complémentarité concevoir entre les évaluations internes et externes? Quelles sont les influences (négatives, positives) entre elles? Quels sont les apports propres à chaque démarche? Dans quelle mesure peuvent-elles cohabiter?

\title{
Conclusion
}

Nous avons vu comment pouvaient se construire des problématiques d'évaluation de dispositifs (stratégiques et méthodologiques) et donner lieu, alors, à des investigations spécifiques à un accompagnement et à une amélioration du pilotage des mésostructures comme les établissements de formation.

II a été postulé que le sens d'une évaluation pouvait être influencé par le choix de ses procédures et que celles-ci reflétaient des modèles sous-jacents qui ne demandaient qu'à être discutés par les utilisateurs.

On en a déduit que les évaluateurs ont tout intérêt à les soumettre à analyse et à remise en question car ce sont là autant de leviers pour une politique d'évaluation en gestation.

Nous avons vu, par ailleurs, comment se posaient les problématiques concernant les pratiques d'évaluations internes et externes des apprentissages des élèves: face à "l'approche par compétences ", la question de l'évaluation devait être posée en termes

${ }^{16}$ Composés de questions à choisir (choix alternatif, choix multiple, ...) ou de questions à réponses courtes. 
Dispositif pédagogique? Dispositif didactique? Situations d'apprentissage !

"d'ingénierie didactique " à propos des types de tâches, de contraintes et de ressources externes mises à la disposition des apprenants.

Ceci a donné à penser que de nouvelles représentations de l'évaluation des apprentissages des élèves et de l'interprétation des résultats par les enseignants étaient engagées.

La séparation entre apprentissage et évaluation est alors apparue comme amoindrie, la temporalité de l'évaluation certificative devient plus longue et l'opposition classique entre évaluation certificative et formative est ainsi remise en question.

Mais, alors, la conception des cadres de référence pour les apprentissages, récemment adoptée, avec des évaluations externes normalisées à l'échelle nationale ou régionale, répond-t-elle à ces questionnements ? Ces cadres de références seraient-ils à réexaminer à la lumière des modélisations et des problématiques présentées plus haut ?

Autant de questions ne pouvant qu'aider à préciser la place de programmes de recherche spécifiques dans l'élaboration d'une politique d'évaluation dans le cadre de la réforme éducative marocaine.

\section{Références bibliographiques}

Akrim, H., El-Jamali, S., El-Bousaadani, A., Radid, M., \& Talbi, M. (2006). L'intégration des QCM dans le contrôle continu des étudiants de 1ère année universitaire SMP et SMC. Cas de la Chimie Générale. Revue Africaine de Didactique des Sciences et des Mathématiques, 1 (août 2006). Consulté sur [http://www.mr.refer.org/radisma/].

Allal, L. (2007). Evaluation dans le contexte de l'apprentissage situé : Peut-on concevoir l'évaluation comme un acte de participation à une communauté de pratiques? In M. Behrens (Ed.), La qualité en éducation. Pour réfléchir à la formation de demain (pp. 39-56). Québec : Presses de l'Université du Québec.

Allal, L., \& Schwartz, G. (1996). Quelle place pour l'évaluation formative dans l'enseignement au cycle d'orientation? CO Infos, 178, 5-8.

Altet, M. (1997). Les pédagogies de l'apprentissage. Paris: Presses Universitaires de France.

De Landsheere, G. (1994). Le pilotage des systèmes d'éducation. Bruxelles : De Boeck.

Dierendonck, D., \& Fagnant, A. (2010). Quelques réflexions autour des épreuves d'évaluation développées dans le cadre de l'approche par compétences. Bulletin de l'ADMEE-Europe, 522. Consulté sur [http://www.admee.org/images/docs/Bulletins/ bulletin_2010_1.pdf].

Figari, G. (1994). Evaluer : quel référentiel ? Bruxelles : De Boeck.

Figari, G. (2008). L'évaluation des dispositifs éducatifs. Mesure et évaluation en éducation, 31(3), 77-93.

Figari, G., \& Mottier Lopez, L. (Ed.) (2006). Recherche sur l'évaluation en éducation: problématiques, méthodologies et épistémologie. Paris : L'Harmattan.

Foucault, M. (1994). Dits et écrits. Paris : Gallimard.

Lafontaine, D., Soussi, A., \& Nidegger, C. (2009). Evaluations internationales et/ou épreuves nationales: tensions et changement de pratiques. In L. Mottier Lopez \& M. Crahay (Ed.), Evaluations en tension : entre la régulation des apprentissages et le pilotage des systèmes (pp. 61-80). Bruxelles : De Boeck. 
Hamadi Akrim, Gérard Figari, Lucie Mottier Lopez, Mohammed Talbi

La place de l'évaluation dans la réforme du système éducatif marocain : questions pour la recherche

Maroy, C. (2009). Régulation post-bureaucratique des systèmes d'enseignement et travail enseignant. In L. Mottier Lopez \& M. Crahay (Ed.), Evaluations en tension : entre la régulation des apprentissages et le pilotage des systèmes (pp. 83-100). Bruxelles : De Boeck.

Montandon, C. (2002). Approches systémiques de dispositifs pédagogiques, enjeux et méthodes. Paris : L'Harmattan.

Mottier Lopez, L. (2010). Perspectives conclusives : repenser la complémentarité des évaluations formative et certificative des compétences professionnelles. In L. Bélair, C. Lebel, $\mathrm{N}$. Sorin, \& A. Roy (Ed.), Evaluation et régulation des compétences professionnelles : entre référentiels et pratiques (pp. 301-312). Ottawa : Presses Universitaires d'Ottawa.

Ntamakiliro, L., \& Longchamp, A.-L. (2008). Aspects de la standardisation de l'évaluation externe dans le canton de Vaud. Actes du $20^{\text {ème }}$ colloque de l'ADMEE-Europe. Consulté sur [https://plone2.unige.ch/admee08/symposiums/j-s6/j-s6-2?searchterm=longchamp].

Pallascio, R., \& Beaudry, N. (2000). L'école alternative et la réforme en éducation : continuité ou changement ? Québec : Presses Universitaires du Québec.

Perrenoud, P. (1993). Six façons éprouvées de faire échouer une réforme scolaire. Intervention au colloque "La profession enseignante au temps des réformes ", Université de Montréal, 19-21 novembre 2003.

OCDE, (2008). Politiques et gestion de l'enseignement supérieur. Paris : Editions de l'OCDE. Peeters, H., \& Charlier, P. (1999). Contributions à une théorie du dispositif. Hermès, 25, 1523.

Rey, B., Carette, V., Defrance, A., \& Kahn, S. (2003). Les compétences à l'école. Bruxelles : De Boeck.

Rodrigues, P. (2006). Logiques d'évaluation en relation avec des conceptions élargies du monde et de la connaissance. In G. Figari \& L. Mottier Lopez (Ed.), Recherche sur l'évaluation en éducation: problématiques, méthodologies et épistémologie (pp. 193-201). Paris : L'Harmattan.

Roegiers, X. (2001). Une pédagogie de l'intégration. Compétences et intégration des acquis dans l'enseignement. Bruxelles: De Boeck.

Scallon, G. (2004). L'évaluation des apprentissages dans une approche par compétences. Bruxelles : De Boeck.

Shadish, W. R., Cook, T., \& Leviton, L. C. (1991). Fondations of Program Evaluation. Theories of Practice. Newbury Park: Sage Publications.

Whitehead, A. N. (1929). The Aims of Education \& Other Essays. New York: Macmillan. 